\title{
Strong Recursive Feasibility in Model Predictive Control of Biped Walking
}

\author{
Matteo Ciocca ${ }^{1}$, Pierre-Brice Wieber ${ }^{2}$, Thierry Fraichard ${ }^{1}$
}

\begin{abstract}
Realizing a stable walking motion requires satisfying a set of constraints. Model Predictive Control (MPC) is one of few suitable methods to handle such constraints. The capacity to satisfy constraints, which is usually called feasibility, is classically guaranteed recursively. In our applications, an important aspect is that the MPC scheme has to adapt continuously to the dynamic environment of the robot (e.g. collision avoidance, physical interaction). We aim therefore at guaranteeing recursive feasibility for all possible scenarios, which is called strong recursive feasibility. Recursive feasibility is classically obtained by introducing a terminal constraint at the end of the prediction horizon. Between two standard approaches for legged robot, in our applications we favor a capturable terminal constraint. When the robot is not really planning to stop and considers actually making a new step, recursive feasibility is not guaranteed anymore. We demonstrate numerically that recursive feasibility is actually guaranteed, even when a new step is added in the prediction horizon.
\end{abstract}

\section{INTRODUCTION}

Walking depends on contact forces between the feet and the ground. The unilateral nature [1] of this interaction (the feet can only push on the ground) limits the motion that a legged robot can realize and plays a crucial role in its stability. In the case of walking on a flat ground, this corresponds to having the Center of Pressure $(\mathrm{CoP})$ stay within the support polygon [2]. Realizing a stable walking motion requires satisfying a set of constraints [3]. Model Predictive Control (MPC) is one of few suitable methods to handle such constraints [4]. It has been used therefore extensively for the control of legged robots [5], [6]. For example, the MPC scheme in [7] generates a walking motion online with automatic footstep placement. It was expanded to ensure safe navigation in a crowd [8] and physical collaboration with humans [9].

MPC solves an optimal control problem over a prediction horizon. The solution is a control sequence that satisfies a set of constraints. The first element of the sequence is applied to the system. The whole process is then repeated. The capacity to satisfy constraints, which is usually called feasibility, is classically guaranteed recursively [10]. An important aspect of our applications is that the MPC scheme has to adapt continuously to the dynamic environment of the robot: collision avoidance [8], physical interaction with humans [9], or visual feedback [11]. We aim therefore at

\footnotetext{
1 Univ. Grenoble Alpes, Inria, CNRS, Grenoble INP, LIG, 38000 Grenoble, France matteo.cioccadinria.fr, thierry.fraichardeinria.fr

2 Univ. Grenoble Alpes, Inria, CNRS, Grenoble INP, LJK, 38000 Grenoble, France pierre-brice.wiebereinria.fr
}

guaranteeing recursive feasibility for all possible scenarios, which is called strong recursive feasibility [12].

Recursive feasibility is classically obtained by introducing a terminal constraint at the end of the prediction horizon [10] to make sure the system remains feasible indefinitely after the end of the horizon. Two standard approaches for legged robots are (i) to consider that the robot keeps repeating indefinitely the same cyclic motion [13], [14], [15] or (ii) that it stops after a given number of steps [16], [17], [8], what corresponds to capturability [18], [19]. Passive safety requires that the robot is able to stop before any collision occurs [20]. For that reason we favor a capturable terminal constraint as in [8].

We can see in Fig. 1 how such a capturability terminal constraint makes sure that the system remains feasible indefinitely. This way, when the prediction horizon advances as in Fig. 2, we are sure that the MPC scheme remains feasible: strong recursive feasibility is guaranteed by construction. The problem is when the robot is not really planning to stop, and considers actually making a new step. With such a sudden change, recursive feasibility is not guaranteed anymore, as shown in Fig. 3. It seems that this issue has been overlooked in the literature of MPC for legged robots. Note that this issue is unrelated to the length of the prediction horizon.

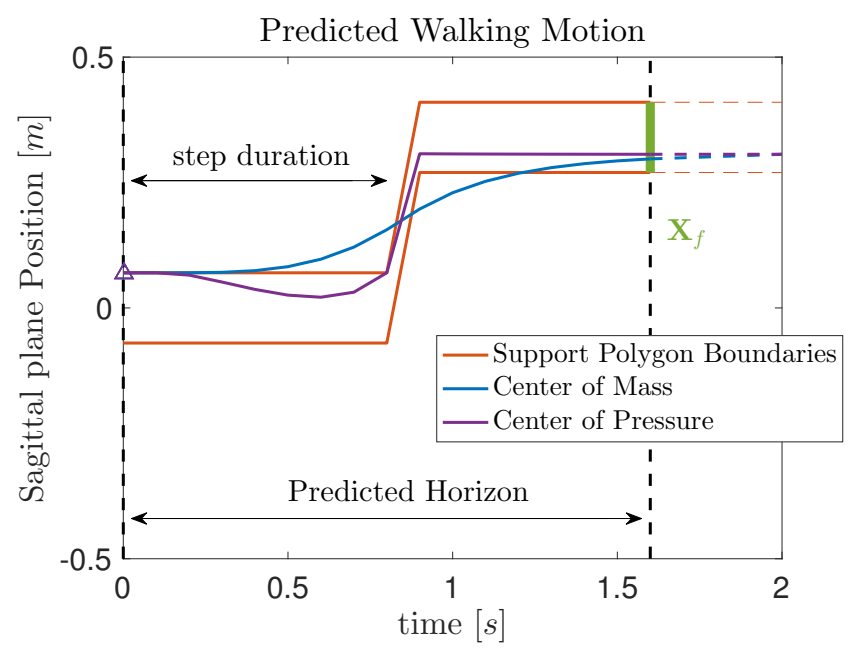

Fig. 1: A capturability terminal constraint (that defines a terminal constraint set $\mathbb{X}_{f}$ ) makes sure that the motion of the legged robot remains feasible indefinitely.

The objective of this paper is to investigate this issue and provide a numerical evidence that recursive feasibility is actually guaranteed, even when a new step is added in the 
prediction horizon.

Outline of the Paper: Section II introduces the definition of strong recursive feasibility in MPC. Section III describes how to provide a numerical evidence of strong recursive feasibility. The dynamics of walking is described in Section IV. Section V describes where we apply our numerical approach. Results of our numerical approach are presented in Section VI.

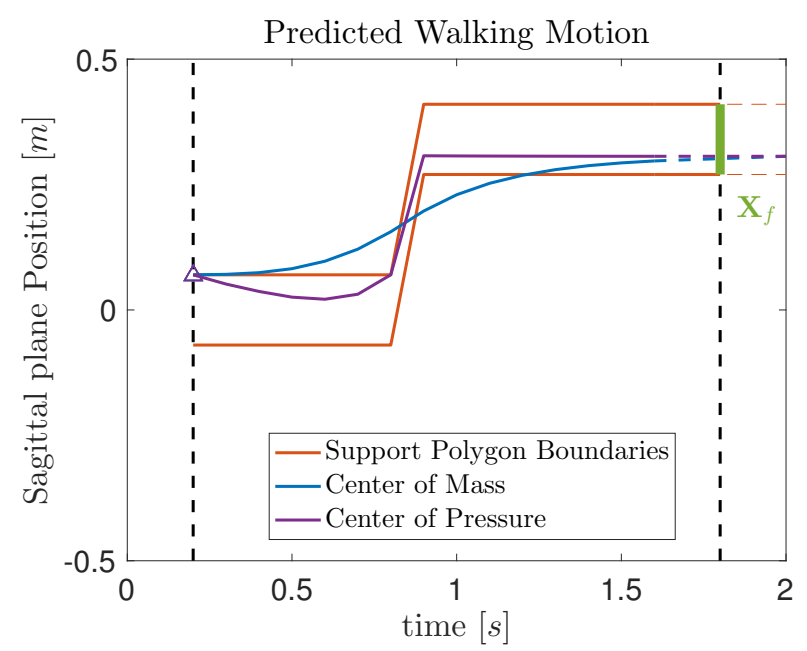

Fig. 2: When the prediction horizon advances, thanks to the fixed terminal constraint set $\mathbb{X}_{f}$, the MPC scheme remains feasible.

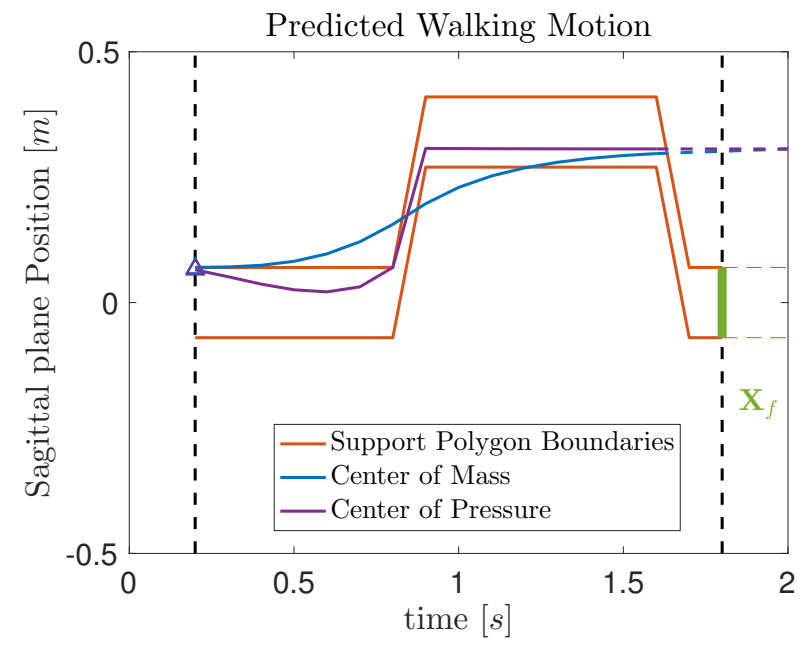

Fig. 3: When the robot considers making a new step, with such a sudden change, recursive feasibility is not guaranteed anymore.

\section{Strong Recursive Feasibility in Model PREDictive CONTROL}

Consider a linear time-invariant discrete-time system

$$
x_{i+1}=A x_{i}+B u_{i},
$$

where $x_{i}, u_{i}$ and $x_{i+1}$ are respectively the state, control and successor state, and $(A, B)$ are matrices of proper dimensions. For a state $x_{i}$, an MPC sheme aims to find a control sequence $\mathcal{U}_{i}=\left\{u_{(i \mid i)}, u_{(i+1 \mid i)}, \ldots, u_{(i+N \mid i)}\right\}^{[1]}$ that satisfies a set of constraints

$$
E_{i} x_{i}+F_{i} \mathcal{U}_{i} \leq d_{i},
$$

where $\left(E_{i}, F_{i}\right)$ are time-varying matrices and $d_{i}$ is a timevarying vector of proper dimensions. Let the set of solutions be

$$
\mathcal{W}_{i} \triangleq\left\{\left(x_{i}, \mathcal{U}_{i}\right) \mid E_{i} x_{i}+F_{i} \mathcal{U}_{i} \leq d_{i}\right\}
$$

An MPC scheme classically chooses a sequence $\mathcal{U}_{i}$ that minimizes an objective function [4], and its first element $u_{(i \mid i)}=\kappa$ is applied to the system. Two useful projections of $\mathcal{W}_{i}$ are therefore:

$$
\left(\mathcal{W}_{\kappa}\right)_{i} \triangleq\left\{\left(x_{i}, \kappa\right) \mid \exists \mathcal{U}_{i} \text { s.t. }\left(x_{i}, \mathcal{U}_{i}\right) \in \mathcal{W}_{i} \wedge u_{(i \mid i)}=\kappa\right\},
$$

and

$$
\mathcal{X}_{i} \triangleq\left\{x_{i} \mid \exists \mathcal{U}_{i} \text { s.t. }\left(x_{i}, \mathcal{U}_{i}\right) \in \mathcal{W}_{i}\right\}
$$

With these sets, we can define strong recursive feasibility in the following way:

Definition 1 (Strong Recursive Feasibility [12]): The MPC scheme is strongly recursive feasible if and only if $\forall i$

$$
\forall\left(x_{i}, \kappa\right) \in\left(\mathcal{W}_{\kappa}\right)_{i}, x_{i+1}=A_{i}, x_{i}+B_{i} \kappa \in \mathcal{X}_{i} .
$$

The sets $\mathcal{W}, \mathcal{W}_{\kappa}$ and $\mathcal{X}$ and Definition 1 are represented in Figs. 4 and 5. By definition, these sets are convex polytopes [21]. In our case, they also happen to be closed. Thanks to these properties, it is sufficient to check property (6) only on the vertices of $\mathcal{W}_{\kappa}$, as can be seen on Fig. 5.

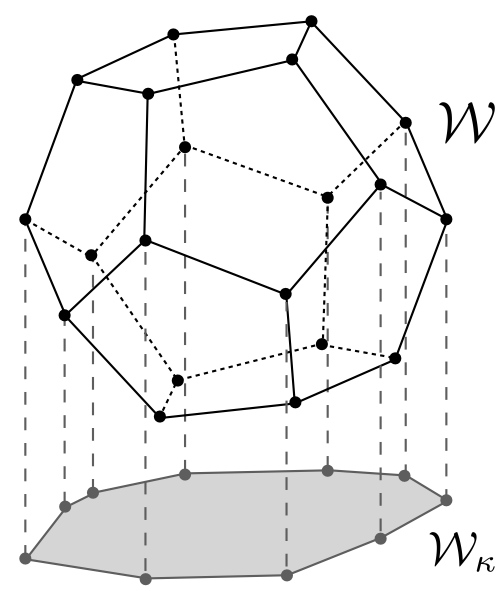

Fig. 4: The polytope $\mathcal{W}$, defined in (3), and its projection $\mathcal{W}_{\kappa}$ defined in (4).

\section{NumericAl Evidence of Strong Recursive FEASIBILITY}

The number of vertices of these polytopes is finite, but enumerating all of them from definition (3) and (4) is

\footnotetext{
${ }^{1}$ the double subscript notation $(i+k \mid i)$ indicates a prediction $k$ steps ahead from time $i$
} 


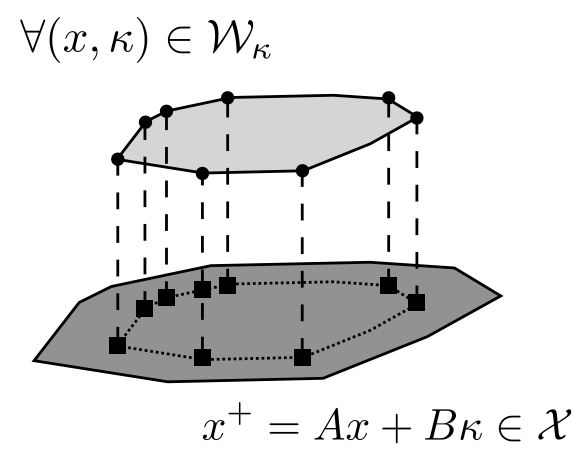

Fig. 5: Strong Recursive Feasibility (Def. 1).

actually an NP-hard problem [22]. Various algorithms and approaches exist, e.g. [23], [24], [25]. We are going to use here a simple randomized approach. The method we propose is based on the observation that the simplex method for Linear Programming (LP) always terminate on a so-called basic solution, which is actually a vertex [26]. We propose therefore to solve LPs of the form

$$
\begin{array}{ll}
\min _{x_{i}, \mathcal{U}_{i}} & \gamma^{T}\left[\begin{array}{l}
x_{i} \\
\mathcal{U}_{i}
\end{array}\right] \\
\text { s.t. } & {\left[\begin{array}{ll}
E_{i} & F_{i}
\end{array}\right]\left[\begin{array}{l}
x_{i} \\
\mathcal{U}_{i}
\end{array}\right] \leq d_{i},}
\end{array}
$$

for randomly chosen directions $\gamma$, which will provide a random selection of vertices of $\mathcal{W}_{i}$. Each vertex found in this way is projected in $\left(\mathcal{W}_{\kappa}\right)_{i}$ and property (6) is checked simply verifying that the following LP

$$
\begin{aligned}
\min _{\mathcal{U}_{i+1}} & \psi^{T} \mathcal{U}_{i+1} \\
\text { s.t. } & E_{i+1}\left(A x_{i}+B \kappa\right)+F_{i+1} \mathcal{U}_{i+1} \leq d_{i+1}
\end{aligned}
$$

has a solution for any direction $\psi$, chosen arbitrarily.

\section{DYNAMICS OF WALKING}

The horizontal motion of the Center of Mass $(\mathrm{CoM}) c \in$ $\mathbb{R}^{2}$ is linearly related to the Center of Pressure (CoP) $p \in \mathbb{R}^{2}$ when walking on a flat ground with constant height [3]:

$$
\ddot{c}=\omega^{2}(c-p)
$$

where $\omega^{2}=g / h, h$ is the height of the CoM above the ground and $g$ is the norm of the gravity vector. This assumes zero rate of change of centroidal angular momentum.

Since contact forces with the ground are unilateral, the CoP is always constrained within the support polygon $\mathcal{P}$ [27]:

$$
p-s_{j} \in \mathcal{P},
$$

where $s_{j} \in \mathbb{R}^{2}$ is the $j^{t h}$ footstep on the ground. The CoM position is constrained to a closed convex region $\mathcal{C}$ due to the maximal leg length of the robot [28]:

$$
c-s_{j} \in \mathcal{C} .
$$

Biped robots should not cross their legs while walking. The position of the $(j+1)^{t h}$ footstep with respect to the position of the $j^{\text {th }}$ footstep is therefore restricted to a region $\mathcal{S}_{j}$ where the legs do not cross:

$$
s_{j+1}-s_{j} \in \mathcal{S}_{j} .
$$

The robot is said to be 0-step capturable [19] when it can stop without having to make any further step. We enforce this situation at the end of the prediction horizon by introducing the following terminal constraint:

$$
\begin{aligned}
& \xi_{N}=p_{N}, \\
& \dot{\xi}_{N}=0
\end{aligned}
$$

where $\xi \in \mathbb{R}^{2}$ is the Capture Point defined as:

$$
\xi=c+\omega^{-1} \dot{c} .
$$

Note that the constraints (10)-(11) and (12) are time varying due to the introduction of the step position $s_{j}$.

\section{Problem Formulation}

In both horizontal coordinates $x$ and $y$, the motion of the CoM $c$ of a legged robot is commonly modeled as a triple integrator [29]:

$$
\hat{c}_{i+1}=\left[\begin{array}{ccc}
1 & T & T^{2} / 2 \\
0 & 1 & T \\
0 & 0 & 1
\end{array}\right] \hat{c}_{i}+\left[\begin{array}{c}
T^{3} / 6 \\
T^{2} / 2 \\
T
\end{array}\right] \dddot{c}_{i},
$$

where $T$ is the sampling time, $\hat{c}_{i}=\left(c_{i}, \dot{c}_{i}, \ddot{c}_{i}\right)$ and $\hat{c}_{i+1}=$ $\left(c_{i+1}, \dot{c}_{i+1}, \ddot{c}_{i+1}\right)$ are two consecutive states, and $\dddot{c}_{i}$ is the CoM jerk. Thus, the CoM motion state is

$$
x_{i}=\left[\begin{array}{c}
\hat{c}_{i}^{x} \\
\hat{c}_{i}^{y}
\end{array}\right] \in \mathbb{R}^{6} .
$$

By evolving the state (16) $N$-times following the dynamics (15), we obtain:

$$
\hat{\mathbf{C}}=\bar{A}_{c} x_{i}+\bar{B}_{c}\left(\mathcal{U}_{\dddot{c}}\right)_{i}
$$

which relates the sequence $\hat{\mathbf{C}} \in \mathbb{R}^{6 N}$ of $N$ CoM position in $x$ and $y$ with a sequence $\mathcal{U}_{\dddot{c}} \in \mathbb{R}^{2 N}$ of $N$ CoM jerk in $x$ and $y$. The same state evolution can be applied to the relation (9):

$$
\hat{\mathbf{P}}=\bar{A}_{p} x_{i}+\bar{B}_{p}\left(\mathcal{U}_{\dddot{c}}\right)_{i}
$$

where $\hat{\mathbf{P}} \in \mathbb{R}^{2 N}$ describes the evolution of the CoP position and $\left(\bar{A}_{c}, \bar{B}_{c}\right)$ and $\left(\bar{A}_{p}, \bar{B}_{p}\right)$ are described in the Appendix VIII. Similar relationship is applied to the selection of the footsteps as

$$
S_{i}=V_{c} s_{c}+V_{f}\left(\mathcal{U}_{s}\right)_{i}
$$

where $s_{c} \in \mathbb{R}^{2}$ is the current footstep on the ground, $\mathcal{U}_{s} \in \mathbb{R}^{2 m}$ is a sequence of future footstep positions and $\left(V_{c}, V_{f}\right)$ are cyclic time-varying matrices (see Appendix VIII) that determine which foot is on the ground at what time. Equations (17)-(18)-(19) are used to formulate the time-varying constraints

$$
(10) \wedge(11) \wedge(12) \wedge(13)
$$


in the linear form as (2) where

$$
\mathcal{U}_{i}=\left[\begin{array}{ll}
(\mathcal{U} \dddot{c})_{i} & \left(\mathcal{U}_{s}\right)_{i}
\end{array}\right]^{T}
$$

The set of constraints is used to investigate a numerical evidence that the recursive feasibility is actually guaranteed during the critical time transition when a new step appears at the end of the predicted horizon. We indicate the discrete time instants before and after the appearance of a new predicted step at the end of the finite horizon respectively $t$ and $t+1$. This critical time transition is shown for time $t$ in Fig. 1 and for $t+1$ in Fig. 3 .

\section{Evaluation of Randomly Selected Vertices}

The parameters of our legged robot were selected accordingly with the kinematics of the robot HRP-2 [30]. The constraints set (10)-(11)-(12) were defined with respect to the current footstep position, which in our case can be chosen arbitrarily: we chose $s_{c}=s_{t}=(0,0)$. The vertices in $\left(\mathcal{W}_{\kappa}\right)_{t}$ were found with the linear programming problem (7) with the set of constraints $\left(E_{t}, F_{t}, d_{t}\right)$. Instead of projecting each solution $\mathcal{W}_{t}$ onto $\left(\mathcal{W}_{\kappa}\right)_{t}$, it was decided to set to zero the entries of $\gamma$ that do not multiply $x_{t}$ or $\kappa$ and to choose randomly the others. Each new vertex was determined to be unique or a duplicate of the ones found before. The finite number of vertices at time $t$ were used to check property (6) with the LP (8) with the set of constraints $\left(E_{t+1}, F_{t+1}, d_{t+1}\right)$.

\section{A. Results}

The choice of robot's model and constraint parameters is summarized in Table I. Three millions vertices were found with (7). Within the set of 3 million vertices, many of them were duplicates. Without repetitions, the total number of vertices was 180 . In Fig. 6 , after $\approx 1.8$ million randomly found vertices, we found only duplicates of the 180 vertices. Thus, the search of the finite number of vertices in $\left(\mathcal{W}_{\kappa}\right)_{t}$ was concluded. Successively, the LP (8) had a solution for all 180 vertices with the constraints at time $t+1$. MATLAB $\mathrm{R} 2016$ a was used to run both linear programming problems (7)-(8) with the linprog function (simplex method).

TABLE I: Robot's Parameters

\begin{tabular}{cccc}
\hline Parameter & Symbol & Value & Unit \\
\hline Height of the CoM & $h$ & 0.8 & {$[\mathrm{~m}]$} \\
Feet dimesions & $\left(f_{l}, f_{w}\right)$ & $(0.24,0.14)$ & {$[\mathrm{m}]$} \\
Leg length & $L$ & 0.3 & {$[\mathrm{~m}]$} \\
Feet separation & $\delta$ & 0.2 & {$[\mathrm{~m}]$} \\
Step duration & $t_{s}$ & 0.8 & {$[s]$} \\
Finite Horizon Dimension & $N$ & 16 & - \\
Number of predicted steps & $m$ & 2 & - \\
Sampling time & $T$ & 0.1 & {$[s]$} \\
\hline
\end{tabular}

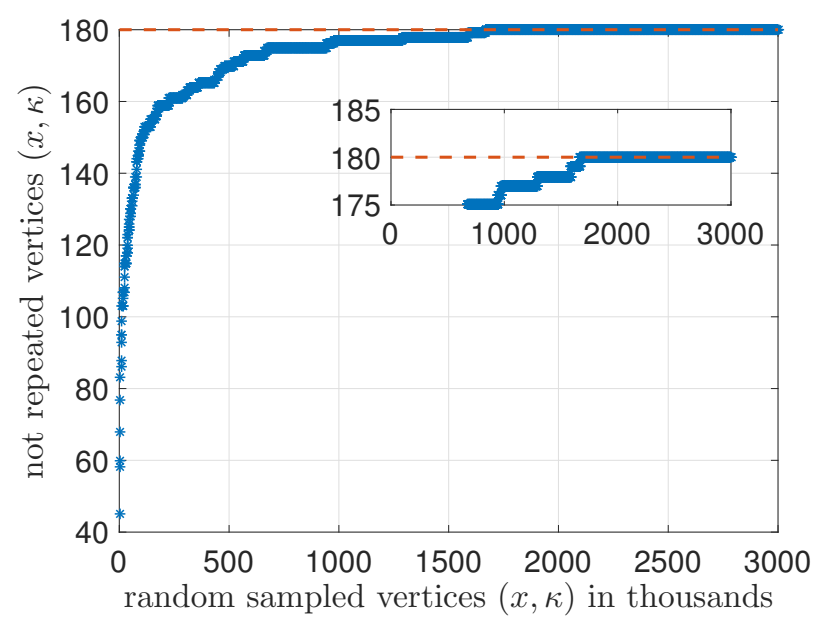

Fig. 6: In a set of 3 million randomly selected vertices $\left(x_{t}, \kappa\right) \in\left(\mathcal{W}_{\kappa}\right)_{t}$, only 180 were unique.

\section{CONCLUSIONS}

A terminal constraint at the end of the prediction horizon guarantees recursive feasibility [10]. We favor a capturable terminal constraint to ensure passive safety of the robot in a dynamic environment [8]. In the case we do not want the robot to stop, we have to change the capturable terminal constraint region periodically in order to consider new steps. And with such sudden change of the terminal constraint, recursive feasibility is not guaranteed anymore.

In this paper we proposed a numerical evidence that shows recursive feasibility is actually guaranteed even when a new step is added in the prediction horizon. In our case the set of constraints for walking is linear and defines a close convex polytope, Section IV. Thanks to these properties we verified property (6) only for the vertices of the polytope when a new step is added in the prediction horizon. Despite the sudden change of the terminal region set, by construction the MPC for legged robot is actually strongly recursive feasible.

Aspects that can lead to further research are: (i) compare our method with one of the algorithms in the literature, e.g. the double description method [24], (ii) investigate whether the MPC scheme loses the strong recursive feasible property due to an inappropriate choice of one (or more) of these three parameters: the length of the finite horizon $N$, the number of predicted steps $m$ or the duration of each predicted step $t_{s}$. For example, the MPC problem could fail to find a solution when the change of the terminal constraint region is close to the present time ( small $N$ ) or when it is suddenly on the next predicted step $(m=1)$.

\section{APPENDIX}

From Section $\mathrm{V}$, the matrices $\left(\bar{A}_{c}, \bar{B}_{c}\right)$ are:

$$
\bar{A}_{c}=\left[\begin{array}{c}
A \\
A^{2} \\
\vdots \\
A^{N}
\end{array}\right], \bar{B}_{c}=\left[\begin{array}{cccc}
B & 0 & \ldots & 0 \\
A B & B & \ldots & 0 \\
\vdots & \vdots & \ddots & \vdots \\
A^{N-1} B & A^{N-2} B & \ldots & B
\end{array}\right]
$$


and $\left(\bar{A}_{p}, \bar{B}_{p}\right)$ are:

$$
\bar{A}_{p}=\left[\begin{array}{c}
C A \\
C A^{2} \\
\vdots \\
C A^{N}
\end{array}\right], \bar{B}_{p}=\left[\begin{array}{cccc}
C B & 0 & \ldots & 0 \\
C A B & C B & \ldots & 0 \\
\vdots & \vdots & \ddots & \vdots \\
C A^{N-1} B & C A^{N-2} B & \ldots & C B
\end{array}\right]
$$

where

$$
\begin{gathered}
A=\left[\begin{array}{cc}
A_{0} & 0 \\
0 & A_{0}
\end{array}\right], A_{0}=\left[\begin{array}{ccc}
1 & T & T^{2} / 2 \\
0 & 1 & T \\
0 & 0 & 1
\end{array}\right] ; \\
B=\left[\begin{array}{c}
B_{0} \\
B_{0}
\end{array}\right], B_{0}=\left[\begin{array}{c}
T^{3} / 6 \\
T^{2} / 2 \\
T
\end{array}\right]
\end{gathered}
$$

and

$$
C=\left[\begin{array}{cc}
C_{0} & 0 \\
0 & C_{0}
\end{array}\right], C_{0}=\left[\begin{array}{lll}
1 & 0 & -\frac{1}{\omega^{2}}
\end{array}\right] \text {. }
$$

And

$$
V_{0}=\left[\begin{array}{c}
1 \\
\vdots \\
1 \\
0 \\
\vdots \\
0 \\
0 \\
\vdots \\
0
\end{array}\right] \in \mathbb{R}^{N \times 1}, V_{1}=\left[\begin{array}{ccc}
0 & 0 & \\
\vdots & \vdots \\
0 & 0 & \\
1 & 0 \\
\vdots & \vdots \\
1 & 0 & \\
0 & 1 & \\
\vdots & \vdots & \\
0 & 1 & \ddots
\end{array}\right] \in \mathbb{R}^{N \times m}
$$

are cyclic time-varying matrices that compose $\left(V_{c}, V_{f}\right)$ as follows:

$$
V_{c}=\left[\begin{array}{cc}
V_{0} & 0 \\
0 & V_{0}
\end{array}\right], V_{f}=\left[\begin{array}{cc}
V_{1} & 0 \\
0 & V_{1}
\end{array}\right]
$$

in which the ones determine which foot is on the ground at what time [2].

\section{ACKNOWLEDGMENT}

This work has been partially supported by the LabEx PERSYVAL-Lab (ANR-11-LABX-0025-01), PSPC Romeo 2 project and EU H2020 Comanoid Research and Innovation Action (RIA). The authors would like to thank Stéphane Caron for the fruitful discussions.

\section{REFERENCES}

[1] P.-B. Wieber, "Viability and predictive control for safe locomotion," in 2008 IEEE/RSJ International Conference on Intelligent Robots and Systems. IEEE, sep 2008. [Online]. Available: https://doi.org/ 10.1109/iros.2008.4651022

[2] A. Herdt, N. Perrin, and P.-B. Wieber, "Walking without thinking about it," in 2010 IEEE/RSJ International Conference on Intelligent Robots and Systems. IEEE, oct 2010. [Online]. Available: https://doi.org/10.1109/iros.2010.5654429

[3] P.-B. Wieber, R. Tedrake, and S. Kuindersma, "Modeling and control of legged robots," in Springer Handbook of Robotics. Springer, 2016, pp. 1203-1234.

[4] D. Mayne, J. Rawlings, C. Rao, and P. Scokaert, "Constrained model predictive control: Stability and optimality," Automatica, vol. 36, no. 6, pp. 789-814, jun 2000. [Online]. Available: https://doi.org/10.1016/s0005-1098(99)00214-9
[5] P.-B. Wieber, "Model predictive control," in Humanoid Robotics. Springer Nature.

[6] D. Gouaillier, C. Collette, and C. Kilner, "Omni-directional closedloop walk for NAO," in 2010 10th IEEE-RAS International Conference on Humanoid Robots. IEEE, dec 2010. [Online]. Available: https://doi.org/10.1109/ichr.2010.5686291

[7] A. Herdt, H. Diedam, P.-B. Wieber, D. Dimitrov, K. Mombaur, and M. Diehl, "Online walking motion generation with automatic footstep placement," Advanced Robotics, vol. 24, no. 5-6, pp. 719-737, jan 2010. [Online]. Available: https://doi.org/10.1163/016918610x493552

[8] N. Bohorquez, A. Sherikov, D. Dimitrov, and P.-B. Wieber, "Safe navigation strategies for a biped robot walking in a crowd," in 2016 IEEE-RAS 16th International Conference on Humanoid Robots (Humanoids). IEEE, nov 2016. [Online]. Available: https://doi.org/10.1109/humanoids.2016.7803304

[9] D. J. Agravante, A. Sherikov, P.-B. Wieber, A. Cherubini, and A. Kheddar, "Walking pattern generators designed for physical collaboration," in 2016 IEEE International Conference on Robotics and Automation (ICRA). IEEE, may 2016. [Online]. Available: https://doi.org/10.1109/icra.2016.7487296

[10] D. Q. Mayne, "Model predictive control: Recent developments and future promise," Automatica, vol. 50, no. 12, pp. 2967-2986, dec 2014. [Online]. Available: https://doi.org/10.1016/j.automatica.2014.10.128

[11] C. Dune, A. Herdt, E. Marchand, O. Stasse, P.-B. Wieber, and E. Yoshida, "Vision based control for Humanoid robots," in IROS Workshop on Visual Control of Mobile Robots (ViCoMoR), San Francisco, USA, United States, Sept. 2011, pp. 19-26. [Online]. Available: https://hal.inria.fr/hal-00639681

[12] E. C. Kerrigan, "Robust constraint satisfaction: Invariant sets and predictive control," Ph.D. dissertation, University of Cambridge, 2001.

[13] T. Takenaka, T. Matsumoto, and T. Yoshiike, "Real time motion generation and control for biped robot: Walking gait pattern generation," in 2009 IEEE/RSJ International Conference on Intelligent Robots and Systems. IEEE, oct 2009. [Online]. Available: https://doi.org/10.1109/iros.2009.5354662

[14] R. Tajima, D. Honda, and K. Suga, "Fast running experiments involving a humanoid robot," in 2009 IEEE International Conference on Robotics and Automation. IEEE, may 2009. [Online]. Available: https://doi.org/10.1109/robot.2009.5152404

[15] K. Nagasaka, Y. Kuroki, S. Suzuki, Y. Itoh, and J. Yamaguchi, "Integrated motion control for walking, jumping and running on a small bipedal entertainment robot," in IEEE International Conference on Robotics and Automation, 2004. Proceedings. IEEE, 2004. [Online]. Available: https://doi.org/10.1109/robot.2004.1308745

[16] A. Takanishi, M. Tochizawa, H. Karaki, and I. Kato, "Dynamic biped walking stabilized with optimal trunk and waist motion," in Proceedings. IEEE/RSJ International Workshop on Intelligent Robots and Systems. IEEE. [Online]. Available: https://doi.org/10.1109/iros. 1989.637905

[17] H. ok Lim, Y. Kaneshima, and A. Takanishi, "Online walking pattern generation for biped humanoid robot with trunk," in Proceedings 2002 IEEE International Conference on Robotics and Automation (Cat. No.02CH37292). IEEE. [Online]. Available: https://doi.org/10.1109/robot.2002.1013705

[18] J. Pratt, J. Carff, S. Drakunov, and A. Goswami, "Capture point: A step toward humanoid push recovery," in 2006 6th IEEE-RAS International Conference on Humanoid Robots. IEEE, dec 2006. [Online]. Available: https://doi.org/10.1109/ichr.2006.321385

[19] T. Koolen, T. de Boer, J. Rebula, A. Goswami, and J. Pratt, "Capturability-based analysis and control of legged locomotion, part 1: Theory and application to three simple gait models," The International Journal of Robotics Research, vol. 31, no. 9, pp. 1094-1113, aug 2012. [Online]. Available: https://doi.org/10.1177/0278364912452673

[20] S. Bouraine, T. Fraichard, and H. Salhi, "Provably safe navigation for mobile robots with limited field-of-views in dynamic environments," Autonomous Robots, vol. 32, no. 3, pp. 267-283, nov 2011. [Online]. Available: https://doi.org/10.1007/s10514-011-9258-8

[21] S. Boyd and L. Vandenberghe, Convex optimization. Cambridge university press, 2004.

[22] J. M. Borwein and P. B. Borwein, Pi and the AGM: A Study in Analytic Number Theory and Computational Complexity. Wiley, New York, 1987.

[23] J. Löfberg, "Oops! I cannot do it again: Testing for recursive feasibility in MPC," Automatica, vol. 48, no. 3, pp. 550-555, mar 2012 [Online]. Available: https://doi.org/10.1016/j.automatica.2011.12.003 
[24] K. Fukuda and A. Prodon, "Double description method revisited," in Combinatorics and Computer Science. Springer Berlin Heidelberg, 1996, pp. 91-111. [Online]. Available: https://doi.org/10.1007/ 3-540-61576-8_77

[25] C. Jones, E. C. Kerrigan, and J. Maciejowski, "Equality set projection: A new algorithm for the projection of polytopes in halfspace representation," Cambridge University Engineering Dept, Tech. Rep., 2004.

[26] D. Bertsimas and J. N. Tsitsiklis, Introduction to linear optimization. Athena Scientific Belmont, MA, 1997, vol. 6.

[27] P.-B. Wieber, "On the stability of walking systems," in Proceedings of the international workshop on humanoid and human friendly robotics, 2002.

[28] C. Brasseur, A. Sherikov, C. Collette, D. Dimitrov, and P.-B. Wieber, "A robust linear MPC approach to online generation of $3 \mathrm{~d}$ biped walking motion," in 2015 IEEE-RAS 15th International Conference on Humanoid Robots (Humanoids). IEEE, nov 2015. [Online]. Available: https://doi.org/10.1109/humanoids.2015.7363423

[29] P.-B. Wieber, "Trajectory free linear model predictive control for stable walking in the presence of strong perturbations," in 2006 6th IEEE-RAS International Conference on Humanoid Robots. IEEE, dec 2006. [Online]. Available: https://doi.org/10.1109/ichr.2006.321375

[30] K. Kaneko, F. Kanehiro, S. Kajita, K. Yokoyama, K. Akachi, T. Kawasaki, S. Ota, and T. Isozumi, "Design of prototype humanoid robotics platform for HRP," in IEEE/RSJ International Conference on Intelligent Robots and System. IEEE. [Online]. Available: https://doi.org/10.1109/irds.2002.1041632 